Check for updates

Cite this: RSC Adv., 2019, 9, 31078

Received 3rd July 2019

Accepted 1st September 2019

DOI: $10.1039 / c 9 r a 05025 f$

rsc.li/rsc-advances

\section{In vitro study of chitosan-based multi-responsive hydrogels as drug release vehicles: a preclinical study}

\author{
Nafisa Gull, (D) *a Shahzad Maqsood Khan, ${ }^{\text {*a }}$ Muhammad Taqi Zahid Butt, ${ }^{\text {b }}$ \\ Syed Khalid, ${ }^{c}$ Muhammad Shafiq, ${ }^{a}$ Atif Islam, ${ }^{a}$ Sumreen Asim, ${ }^{d}$ Sadaf Hafeez ${ }^{a}$ \\ and Rafi Ullah Khan ${ }^{a}$
}

Systematic administration of painkillers and anti-inflammatory drugs is routinely employed to minimize pain and bodily disorders. Controlled drug delivery has the potential to improve the outcomes of disorders by providing sustained exposure to efficacious drug concentrations. Herein, we report the fabrication of multi-responsive hydrogels using reactive and functional polymers such as chitosan and polyvinyl pyrrolidone by varying the concentration of a cleavable crosslinker, tetraethyl orthosilicate. The swelling indices of the hydrogels were evaluated in distilled water, solutions with different $\mathrm{pH}$ values and different electrolytes. FTIR, WAXRD and TGA were conducted to investigate the structures, crystallinities and thermal stabilities of the prepared multi-responsive hydrogels, respectively. The ultimate tensile strength and elongations at break of the fabricated hydrogels were investigated to assess their mechanical stability. Optical microscopy, biodegradation, antimicrobial and cytotoxicity analyses were further carried out to verify the magnified crosslinked and porous structures, biodegradabilities, biocompatibilities and toxic behaviour of the as-prepared hydrogels, respectively. Drug release analysis was conducted to evaluate their release behaviour in PBS, SGF, SIF and electrolyte solutions. The overall results indicate the successful development of novel, non-toxic and sustained drug deliverable hydrogels, which can be considered as a paramount success towards the fabrication of controlled drug delivery systems.

\section{Introduction}

The notion of controlled drug release was initiated by the demand for effectual treatment of diseases. ${ }^{1}$ Targeted controlled release systems proffer several distinguishing benefits over conventional drug delivery methods. These include targeted delivery of the drug to a specific part of the body, drug stability, pledge of treatment continuity in nocturnal phase, optimal drug absorption and less need of follow-up care. Controlled release systems have been reported over a range of $\mathrm{pH}$ domains in the body, e.g. for intestinal, gastric, oral and periodontal applications. ${ }^{2-4}$ Several studies have been reported for the use of hydrogels as controlled release systems. ${ }^{5}$

Multi-responsive hydrogels (MRHs) are 3-D polymeric networks that swell in the presence of different stimuli, such as

${ }^{a}$ Department of Polymer Engineering and Technology, University of the Punjab, Lahore, 54590, Pakistan.E-mail: nafisagull@gmail.com; shahzadmqkhan@hotmail.com; shahzad.pet.ceet@pu.edu.pk; Tel: +92 333897 6303; +92 3007152120

${ }^{b}$ College of Engineering and Emerging Technology, University of the Punjab, Lahore, 54590, Pakistan

${ }^{C}$ Research Center of Materials Science, Beijing Institute of Technology, Beijing 100081, P. R. China

${ }^{d}$ Khwaja Fareed University of Engineering and Information Technology, Rahim Yar Khan, 64200, Pakistan aqueous medium, physiological $\mathrm{pH}$, temperature, and ionic solutions. Due to their high water content, hydrogels have potential biomedical applications, such as in contact lenses, wound healing, tissue substitutes and slow drug delivery systems; they have been proved to be biocompatible and to enhance tissue regeneration and cell growth. ${ }^{6-10}$ Copolymer or multipolymer hydrogels are smart materials because a variety of physical and chemical properties for biomedical applications can be induced by joining multiple monomeric units. ${ }^{11}$ Natural functional and reactive polymers such as chitosan (CS), chitin, guar gum and alginate are used for drug delivery systems because they can play vital role in healing procedures. However, the use of natural polymers is limited because of their poor mechanical properties and difficult processing. The combination of reactive synthetic and natural polymers can endow a drug delivery system with optimum properties. ${ }^{12}$

CS is a natural cationic biopolymer that is produced by $\mathrm{N}$ deacetylation of chitin, the second most abundant natural polymer after cellulose. It is an interesting functional biomaterial due to its good biodegradability, biocompatibility, low cost and low toxicity. ${ }^{\mathbf{1 3 , 1 4}} \mathrm{CS}$ has been broadly used for an extensive range of applications, such as wound-healing agent, drug carriers, tissue engineering, skin regeneration, ${ }^{\mathbf{1 5}}$ membrane filters for water treatment, chelating agents, and 
biodegradable films or coatings for foodstuff packaging. ${ }^{16}$ Polyvinyl pyrrolidone (PVP) is a water-soluble, highly amorphous polymer. It is considered to be a "miracle" polymer because of its numerous distinctive applications. It is used in the fabrication of thromboresistant hydrophilic gels. ${ }^{17}$ Due to the lubricating and viscous properties of PVP, it is useful for vitreous humor replacement. ${ }^{18}$ Both polymers are frequently used in the field of biomaterials, especially for the development of next generation hydrogels. ${ }^{19}$

Because CS is soluble in acidic solvents, therefore its gels are frequently crosslinked to increase the stability of their structures. Dialdehyde, epoxy compounds, formaldehyde, glutaraldehyde, etc. are frequently used crosslinking agents. However, all these materials are chemically synthesized and can cause physiological toxicology. ${ }^{20}$ However, tetraethylorthosilicate (TEOS) is a preferred crosslinker because it forms covalent bonds between inorganic regions and amorphous polymer chains. It has been successfully used in biomedical applications and is non-toxic, unlike typically reported crosslinkers such as tripolyphosphate, borate, and glutaraldehyde. ${ }^{21,22}$ A critical review of the reported literature was carried out on MRHs, covering different aspects such as natural and synthetic polymers, types of crosslinker, and modes of fabrication. As per the findings of our extensive literature survey, this study is the first to propose the fabrication of MRHs comprising CS and PVP crosslinked with TEOS by a solution casting protocol for controlled drug release systems.

The foremost issue in typical drug delivery systems is that; normally the drug is not released in significant amount. Therefore, a small concentration is absorbed by the body and the remainder is excreted from the body without any change. When working with drugs which are not easily consumed by the body, it is required to adopt alternative approaches to maintain the drug dosage in the prescribed amount. To address this problem, hydrogels were introduced; they release drugs in a controlled manner with a suitable delivery timespan. ${ }^{23,24}$ Lidocaine was the first modern local anaesthetic agent, and it was used as a model drug in this study. It was selected due to its clinical relevancy in broad spectrum therapy for burning, itching and pain from skin inflammation. ${ }^{25}$

In this study, CS/PVP MRHs were fabricated via a solution casting protocol in the presence of TEOS. The prepared hydrogels were characterized by swelling in distilled water, different $\mathrm{pH}$ media and electrolytes, gel fractions, Fourier transform infrared spectroscopy (FTIR), optical microscopy, Xray diffraction spectroscopy, and thermogravimetric analysis to determine their thermal stability, cytotoxicity, antimicrobial analysis and drug release in phosphate buffer saline (PBS), simulated gastric fluid (SGF), simulated intestinal fluid (SIF) and electrolyte solutions.

\section{Experimental details}

\subsection{Materials}

CS (molecular weight $90000 \mathrm{~g} \mathrm{~mol}^{-1}$; DDA 90\%) was procured from Germany. PVP (molecular weight $40000 \mathrm{~g} \mathrm{~mol}^{-1}$ ), formic acid ( $\geq 96 \%$ ) and TEOS were purchased from Sigma Aldrich, USA. Boric acid, calcium chloride and sodium chloride were purchased from Riedel-deHaën, potassium chloride, potassium dihydrogen phosphate and sodium dihydrogen phosphate were purchased from Merck, Germany, and hydrochloric acid (37\% pure) was obtained from Riedel-deHaën. All chemicals were of analytical grade and were used without any further purification.

\subsection{Methods}

Hydrogels were prepared by the economical solution casting technique. CS (60 $\mathrm{wt} \%$, i.e. $0.6 \mathrm{~g}$ ) was dissolved in $2 \%$ formic acid (50 mL), and PVP (40 wt\%, i.e. $0.4 \mathrm{~g}$ ) was dissolved separately in distilled water $(50 \mathrm{~mL})$. The two solutions were mixed and blended for $2 \mathrm{~h}$ at $50{ }^{\circ} \mathrm{C}$ to $55^{\circ} \mathrm{C}$. TEOS $(50 \mu \mathrm{L})$ was taken in $5 \mathrm{~mL}$ of ethanol and added dropwise to the prepared blend, and this mixture was vortexed for an additional $4 \mathrm{~h}$ at the same temperature. Later, the mixture was poured in a Petri dish and
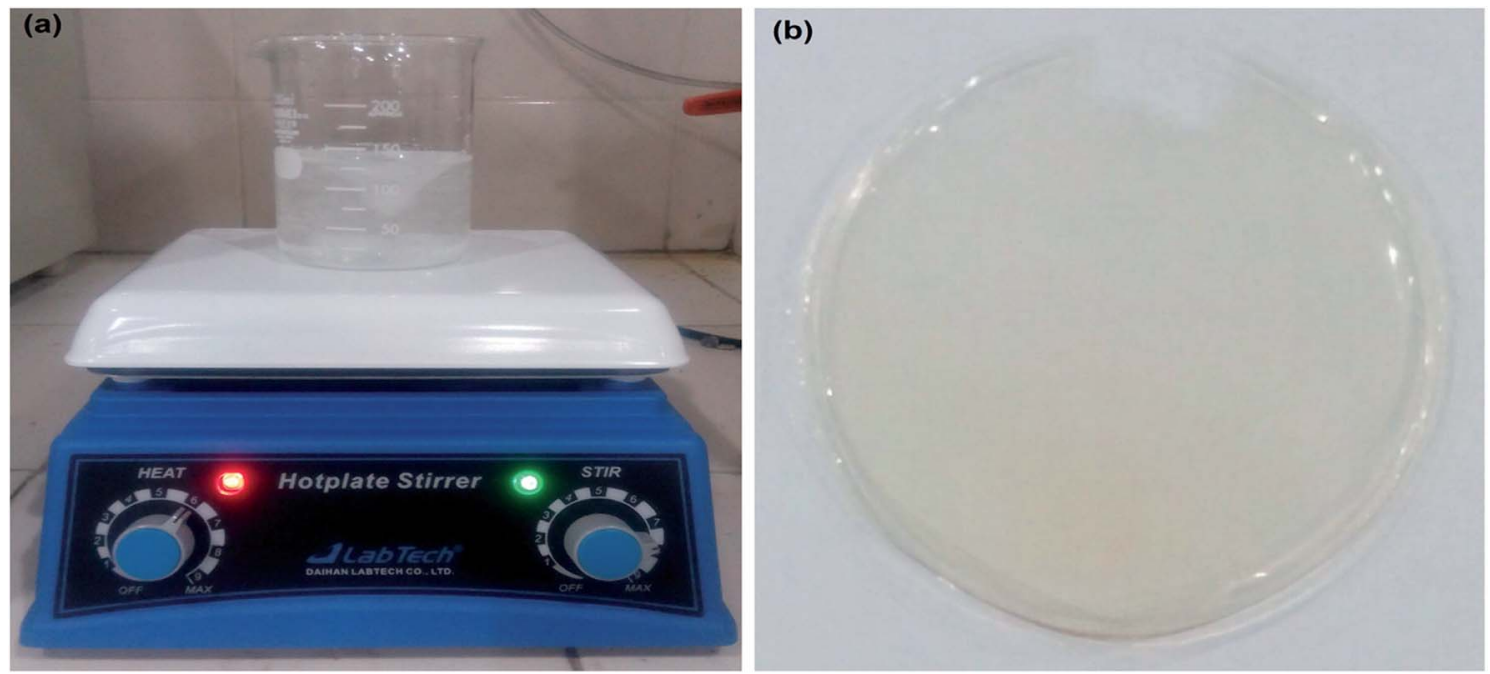

Fig. 1 Digital pictures (a) before hydrogel fabrication; (b) after hydrogel fabrication. 


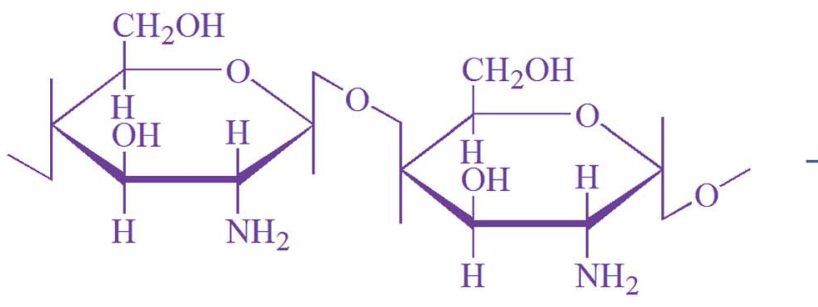<smiles>CCO[Si](OCC)(OCC)OCC</smiles>

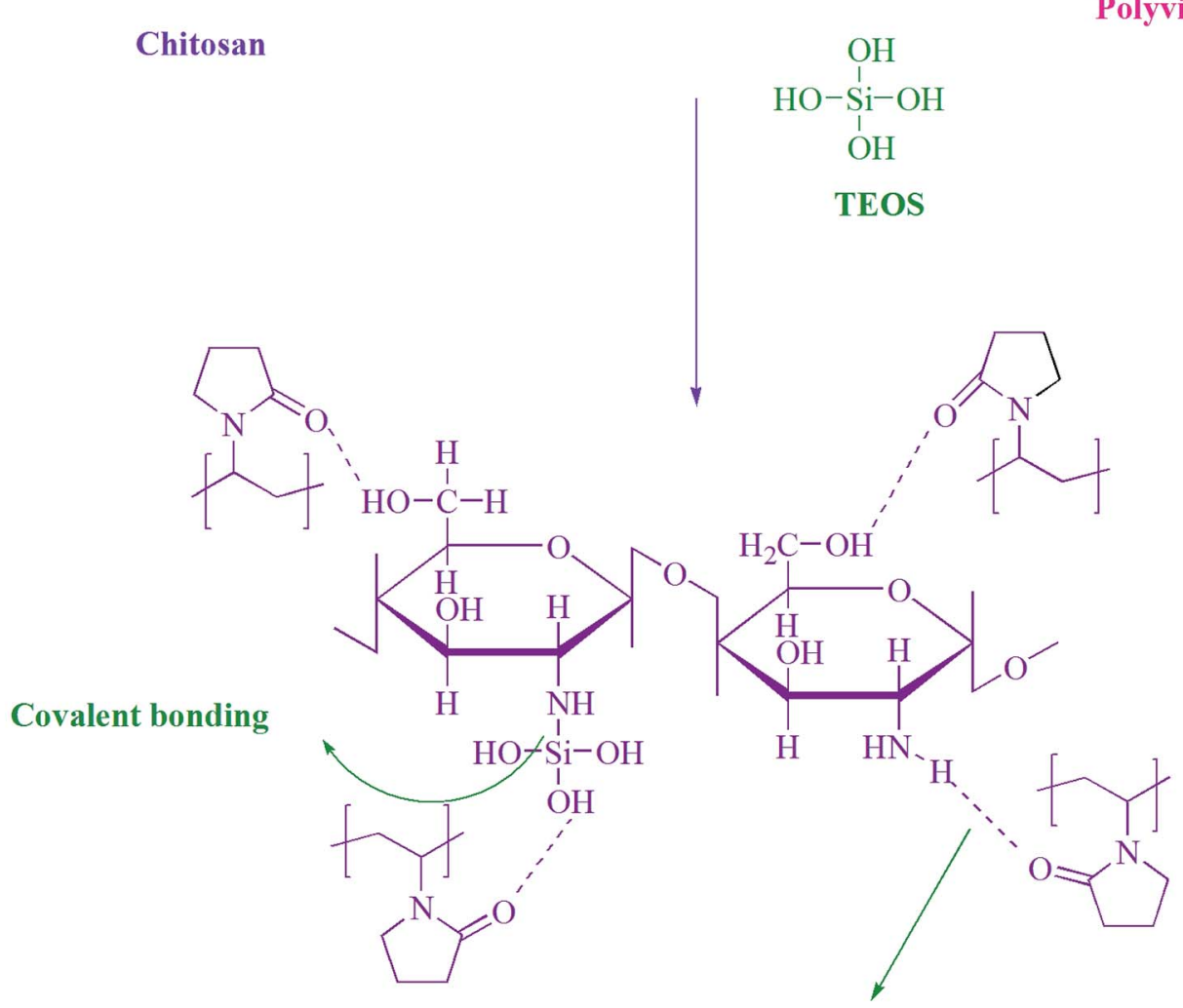

Polyvinylpyrrolidone

H-bonding

Scheme 1 Proposed interactions among the components of the prepared MRHs.

placed in a vacuum drying oven (LVO-2040, Lab Tech, Korea) at $40{ }^{\circ} \mathrm{C}$ until it completely dry; it was then stored at room temperature for further studies. Digital images of the hydrogel mixture before and after development are shown in Fig. 1.

Hydrogel samples with 100, 150, 200 and $250 \mu \mathrm{L}$ TEOS concentrations were synthesized in the same way. Hydrogel samples without crosslinker were also prepared and were coded as MRH. The MRHs with TEOS concentrations of 50, 100, 150, 200 and $250 \mu \mathrm{L}$ were coded as MRH-1, MRH-2, MRH-3, MRH-4 and MRH-5, respectively. The proposed interactions among the components of the prepared hydrogels are shown in Scheme 1.

\subsection{Drug-loaded hydrogels}

CS (0.6 g) was dissolved in $50 \mathrm{~mL}$ of $2 \%$ formic acid solution and PVP ( $0.4 \mathrm{~g})$ was dissolved separately in $50 \mathrm{~mL}$ of distilled water at $50{ }^{\circ} \mathrm{C}$, as discussed in Section 2.2. The two solutions were blended for $2 \mathrm{~h}$ at $50{ }^{\circ} \mathrm{C}$ to $55^{\circ} \mathrm{C}$. Then, $50 \mathrm{mg}$ of model drug (lidocaine) was dissolved in distilled water and was added to the polymer blend; the mixture was stirred for $1 \mathrm{~h} .150 \mu \mathrm{L}$ of TEOS

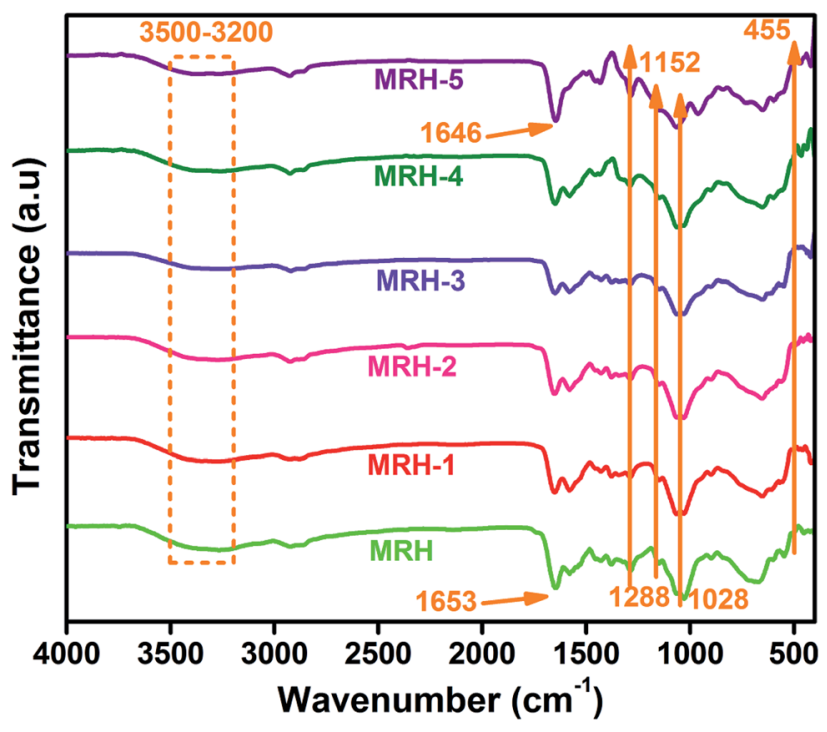

Fig. 2 FTIR spectra of the as-prepared MRHs. 




Fig. 3 Swelling indices of the as-prepared MRHs in distilled water.

was dissolved in $5 \mathrm{~mL}$ of ethanol and was added dropwise to the drug-loaded blend. This blend was further stirred for $4 \mathrm{~h}$ at the same temperature. This solution was then poured in a Petri dish and dried in a vacuum drying oven (LVO-2040, Lab Tech, Korea) at $40{ }^{\circ} \mathrm{C}$. After complete drying, each drug-loaded sample was peeled off the Petri dish and stored at room temperature for the drug release studies.

\section{Characterizations}

\subsection{Fourier transform infrared spectroscopy}

The functional group confirmations and the interactions between all the components of the prepared hydrogels were evaluated by FTIR spectroscopy (Shimadzu, IR Prestige-21, Japan) in attenuated total reflectance (ATR) mode. The analysis was performed in the wavenumber range of 4000 to $400 \mathrm{~cm}^{-1}$ at a scan rate of 60 scans per sample, and the resolution was set as $4 \mathrm{~cm}^{-1}$. The air background was taken before each sample run.

\subsection{Swelling experiments}

3.2.1. Swelling index in distilled water. The swelling indices of the prepared MRHs were determined gravimetrically in distilled water. A small piece of sample was taken and weighed. Then, it was placed in distilled water in a Petri dish and was allowed to swell. After $10 \mathrm{~min}$, the water was removed from the Petri dish, and the sample was cleaned properly with tissue paper and weighed again. This procedure was repeated until the weight of the sample began to decrease or become stable. The swelling indices of the samples were determined using eqn (1):

$$
\text { Swelling index }=\frac{W_{\mathrm{s}}-W_{\mathrm{d}}}{W_{\mathrm{d}}}
$$

where $W_{\mathrm{s}}$ and $W_{\mathrm{d}}$ are the weights of the swollen and dried samples, respectively. The swelling index of each hydrogel was calculated three times, and the average was taken to note the final reading.

3.2.1.1. Gel fraction. To determine the gel fractions, preweighed samples were taken in Petri dishes and immersed in sufficient amounts of distilled water for $10 \mathrm{~h}$. After a specific time duration, the water was removed and the hydrogel samples were dried in a vacuum oven at $40{ }^{\circ} \mathrm{C}$ until the weight become constant. The gel fractions were measured by eqn (2):

$$
\text { Gel fraction }(\%)=\frac{M^{\prime}}{M^{\circ}}
$$

where $M^{\prime}$ refers to the weight of the crosslinked sample after oven drying and $M^{\mathrm{o}}$ is the initial weight of the sample.

3.2.2. Swelling index in buffer solutions. Buffer solutions with different $\mathrm{pH}$ values $(2,4,6,8$ and 10) were prepared by the standard method. Each hydrogel sample was weighed and immersed in buffer solutions till equilibrium time of each sample. All the samples were tested in triplicate. The swelling indices of the hydrogels was measured by the same equation as in Section 3.1.1.

3.2.3. Swelling index in electrolyte solutions. Electrolyte solutions, i.e. $\mathrm{NaCl}$ and $\mathrm{CaCl}_{2}$, with different concentrations 


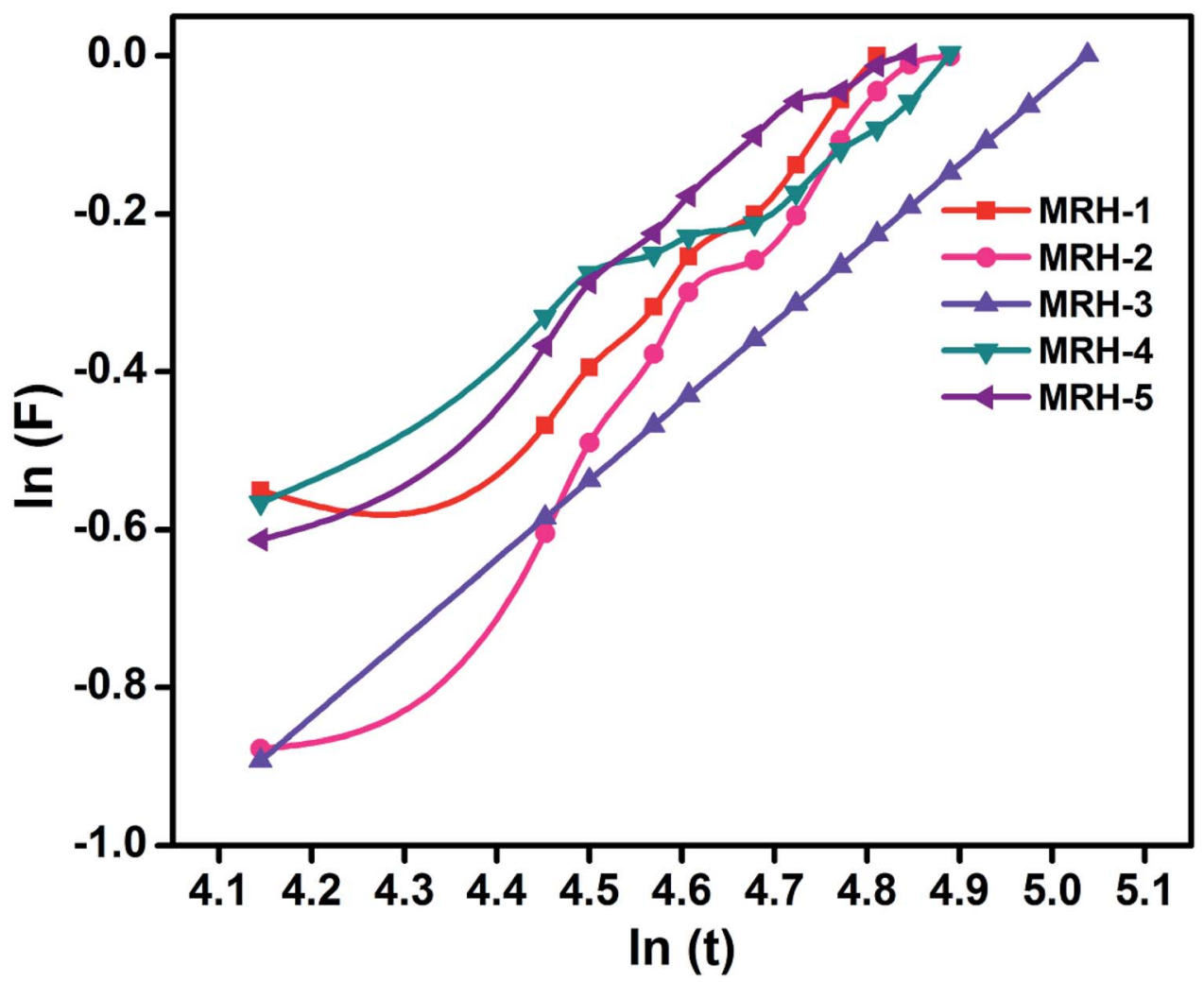

Fig. $4 \ln (F)$ vs. $\ln (t)$ plots of the MRHs.

$(0.1,0.3,0.5,0.7$ and $0.9 \mathrm{M})$ were prepared using the standard method. The swelling indices of the prepared hydrogels in electrolyte solutions were measured by the same procedure as described in Section 3.1.1.

\subsection{Thermogravimetric analysis}

A TGA/STA 409C instrument (NETZSCH, Germany) was used to examine the thermal behaviour of the prepared MRHs. The samples were analyzed from ambient temperature to $650{ }^{\circ} \mathrm{C}$, and the temperature ramp was maintained at $10{ }^{\circ} \mathrm{C} \min ^{-1}$. The samples were tested in flowing argon gas at a flow rate of $15 \mathrm{~mL} \mathrm{~min}{ }^{-1}$.

\subsection{Wide-angle $\mathrm{X}$-ray diffraction analysis}

Wide-angle X-ray diffraction analysis (WAXRD) was carried out to characterize the polymorphic forms of the prepared hydrogels by a Siemens D500 X-ray diffractometer, Germany, equipped with a cobalt source. WAXRD data were collected using an Fe-filtered Co-K $\alpha$ radiation source in the $2 \theta$ range of 5 to $80^{\circ}$ at a scan rate of $0.02^{\circ} \mathrm{s}^{-1}$. The accelerating current and voltage were set at $40 \mathrm{~mA}$ and $40 \mathrm{kV}$, respectively.

\subsection{Mechanical properties}

The mechanical properties of the prepared MRHs were evaluated using a Lloyd LRX material testing machine, UK, equipped with a $200 \mathrm{~N}$ load cell. Samples with dimensions of $2.5 \times 0.5$ inches were used to evaluate the ultimate tensile strength (UTS) and elongation at break (E\%) using the ASTM D882-02 at 20 $\mathrm{mm} \min ^{-1}$ cross head speed of the machine with a gauge length of 1.5 inch at room temperature. These experiments were conducted in triplicate, and the average value of the three samples was calculated to evaluate the mechanical properties.

\subsection{Optical microscopy}

To examine the crosslinking and swelling behaviour of the surface of the MRHs, a research grade polarising microscope (GXM XPL33230; GT Vision, Haverhill, UK) with transmitted and incident polarized light was used. The hydrogel samples were illuminated with monochromatic light to minimize the chromatic deviation, and the images were captured at $20 \times$ magnification.

\subsection{In vitro biodegradation}

To evaluate the in vitro biodegradation of the prepared MRHs, PBS solution was prepared by standard method. Degradation studies were conducted after 1, 3, 5 and 7 days. Pre-weighed

Table 1 Diffusion parameters and gel fractions of the MRHs

\begin{tabular}{lccccc}
\hline Parameter & MRH-1 & MRH-2 & MRH-3 & MRH-4 & MRH-5 \\
\hline$n$ & 0.21 & 0.35 & 0.31 & 0.18 & 0.20 \\
Intercept & -0.466 & -0.741 & -0.691 & -0.406 & -0.434 \\
$K$ & 0.627 & 0.476 & 0.501 & 0.666 & 0.648 \\
Regression $(R \%)$ & 90 & 96 & 98 & 91 & 92 \\
Gel fraction $(\%)$ & 64.68 & 71.23 & 83.37 & 86.24 & 89.65
\end{tabular}


hydrogel samples were immersed in excess amount of PBS solution. After the predetermined time points, the PBS solution was removed carefully and the weight of the degraded sample was measured. The biodegradation of the hydrogel samples was determined by eqn (3).

$$
\text { Biodegradation }=\frac{W_{\mathrm{i}}-W_{\mathrm{d}}}{W_{\mathrm{i}}}
$$

where $W_{\mathrm{i}}$ is the initial weight of the hydrogel and $W_{\mathrm{d}}$ is the degraded weight of the hydrogel.

\subsection{Antimicrobial profile}

The antimicrobial profiles of the MRHs were determined against Escherichia coli (E. coli). Growth of the bacterial strain was checked in sterile Luria-Bertani (LB) medium which was prepared by taking $10 \mathrm{~g}$ Tryptone, $5 \mathrm{~g}$ yeast and $10 \mathrm{~g} \mathrm{NaCl}$ in $800 \mathrm{~mL}$ distilled water. The $\mathrm{pH}$ of the solution was maintained at 7. The solution volume was marked up to $1000 \mathrm{~mL}$ and was sterilized in an autoclave for $1 \mathrm{~h}$. For the control sample, $20 \mu \mathrm{L}$ of $E$. coli strain and $20 \mathrm{~mL}$ of $\mathrm{LB}$ medium were taken in a vessel and incubated for $24 \mathrm{~h}$ at $37^{\circ} \mathrm{C}$. For sample preparation, $20 \mu \mathrm{L}$ E. coli strain and $20 \mathrm{~mL} \mathrm{LB}$ media were mixed; a hydrogel sample $(5 \times 5 \mathrm{~mm})$ was added to the mixture, and the above procedure was repeated. The antimicrobial activity was measured by taking the optical densities of the control and samples with a spectrophotometer (Double beam, PerkinElmer, Model Lambda25, USA) at $600 \mathrm{~nm}$.

\subsection{In vitro cytotoxicity analysis}

In vitro cytotoxicity analysis was performed by the brine shrimp lethality assay protocol, which is a fundamental toxicity screening procedure. Brine shrimps were hatched using brine shrimp eggs in a vessel containing sterile sea water with constant aeration for $48 \mathrm{~h}$ at ambient temperature. After proper hatching, active nauplii were separated from the brighter portion of the hatching vessel and taken for the assay in a deep

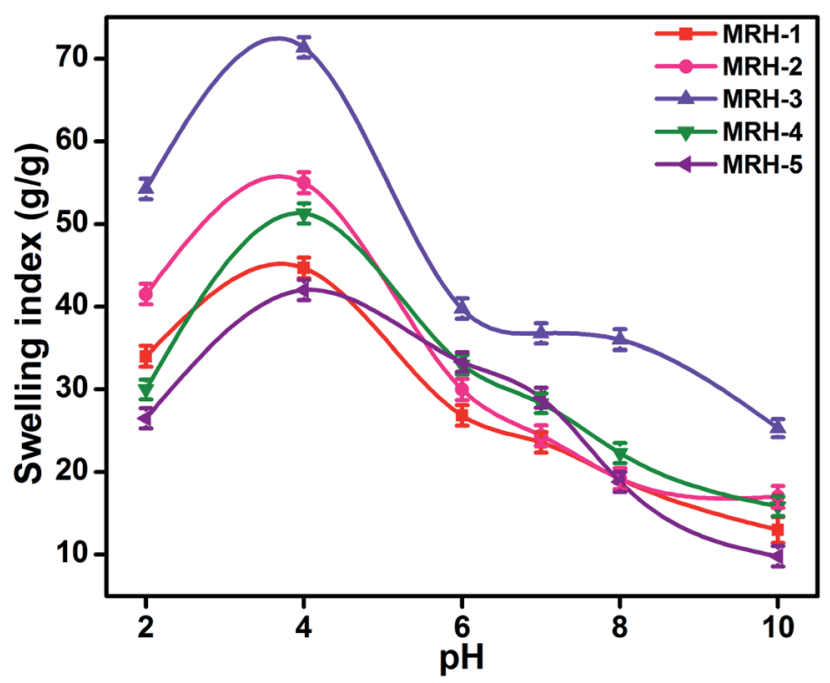

Fig. 5 Swelling indices of the as-prepared MRHs in buffer solutions. well microtitre plate with a well depth of $2 \mathrm{~cm}$ and a well diameter of $1.8 \mathrm{~cm}$ filled with $0.2 \mathrm{~mL}$ salt water. The immature larvae were counted, and hydrogel samples were placed in triplicate in the active nauplii wells. The well plate was kept in the dark at room temperature. Surviving nauplii were observed using a microscope (GXM, XPL33230; GT Vision, Haverhill, UK) and were counted to calculate the mortality using eqn (4):

$$
M(\%)=\frac{A-B-N}{G-N} \times 100
$$

$M=$ percent of dead larvae after $24 \mathrm{~h}, A=$ number of dead larvae after $24 \mathrm{~h}, B=$ average number of dead larvae in the blind samples after $24 \mathrm{~h}, N=$ number of dead larvae before starting the test, $G=$ total number of larvae.

\subsection{Drug release profile}

The drug release profiles were evaluated by submerging the above-prepared drug-loaded hydrogel samples in $100 \mathrm{~mL}$ of PBS solution at physiological conditions ( $\mathrm{pH} 7.4$ and $37^{\circ} \mathrm{C}$ ). This release medium was selected to simulate intestinal fluids and to maintain the $\mathrm{pH}$ of the colon. ${ }^{26}$ After every $10 \mathrm{~min}$, a $5 \mathrm{~mL}$ aliquot of this PBS solution was collected in a vial to check the drug release concentration, and $5 \mathrm{~mL}$ of fresh PBS was added to maintain the constant volume of solution. This process was repeated for $3 \mathrm{~h}$, and the drug release concentration was quantified by measuring the absorbance of the collected sample by UV-Vis analysis (UV-Vis spectrophotometer, double beam, model Lambda 25, PerkinElmer, USA) at $263 \mathrm{~nm}$.

The same procedure was repeated to check the drug release in simulated gastric fluid (SGF; $\mathrm{pH}$ 1.2) and simulated intestinal fluid (SIF; pH 6.8) solutions as well as in electrolyte solutions, i.e. $\mathrm{NaCl}$ and $\mathrm{CaCl}_{2}$.

\section{Results and discussion}

\subsection{Fourier transform infrared spectroscopy}

FTIR is a very useful technique to evaluate chemical changes at the molecular level. The intensity and position of the spectral

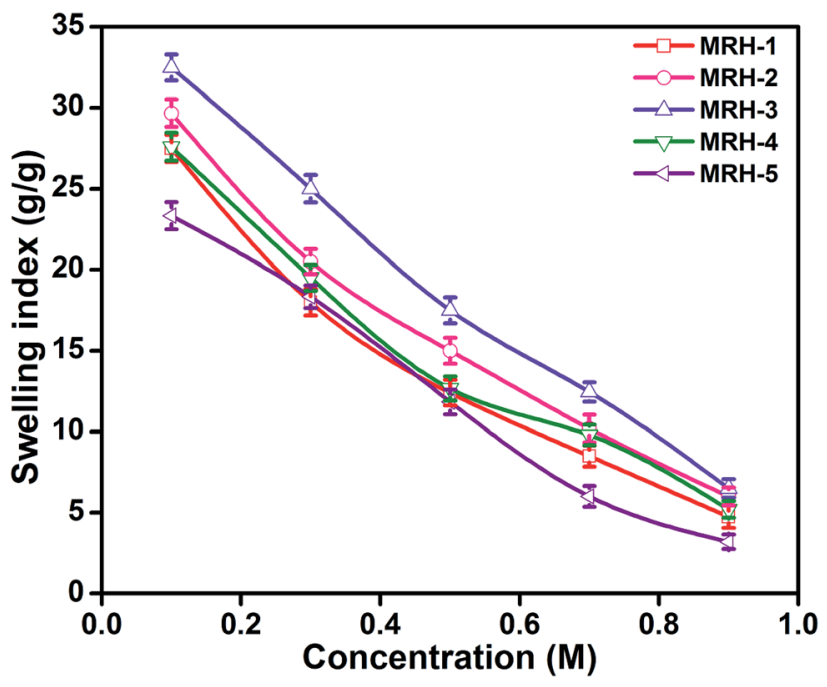

Fig. 6 Swelling indices of the as-prepared $\mathrm{MRH}$ s in $\mathrm{NaCl}$ solutions. 


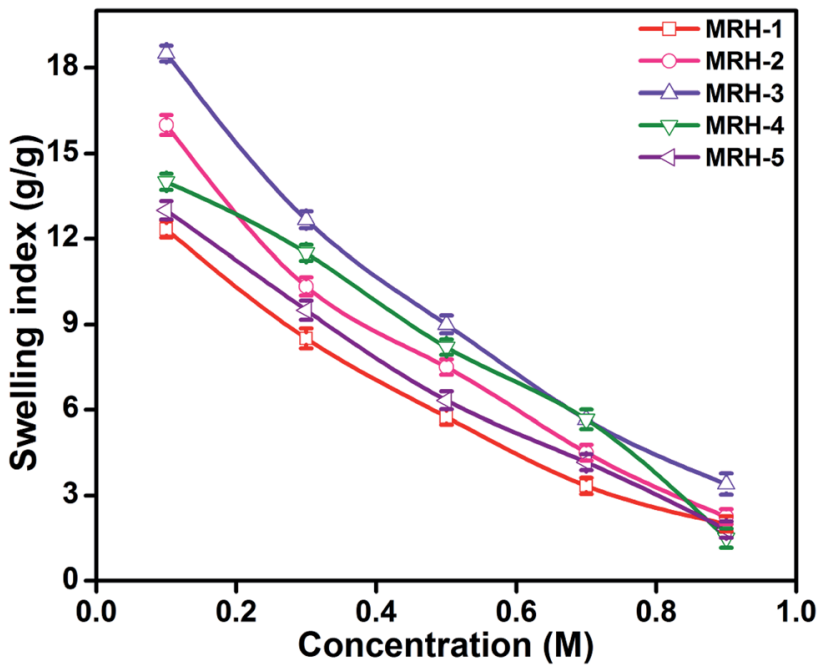

Fig. 7 Swelling indices of the as-prepared $\mathrm{MRH}$ in $\mathrm{CaCl}_{2}$ solutions.

bands are sensitive to chemical changes and provide confirmation of the formation of macromolecules. ${ }^{27}$ This analysis confirms the functional groups present in ingredients and shows intermolecular hydrogen bonding between components because particular interactions influence the local electron density and corresponding frequency shifts. Fig. 2 shows the FTIR spectra of the prepared hydrogel samples. All spectra showed absorption bands in the region of 3500 to $3200 \mathrm{~cm}^{-1}$ corresponded to the - $\mathrm{NH}$ group of $\mathrm{CS}$, which are masked by the absorption bands of the -OH groups present in CS. The bands at 2925 and $2855 \mathrm{~cm}^{-1}$ are assigned to asymmetric and symmetric vibrations of $-\mathrm{CH}$ bonds, respectively. ${ }^{19}$ The existence of hydrogen bonding between components is inferred by the shift of the sharp band at $1653 \mathrm{~cm}^{-1}$ towards a lower wave number $\left(1646 \mathrm{~cm}^{-1}\right)$. This hydrogen bonding may occur between the carbonyl groups of PVP and the hydroxyl groups of $\mathrm{CS},{ }^{28}$ as shown in Scheme 1. The new $\mathrm{Si}-\mathrm{N}$ bonds which develop due to the interactions between CS and TEOS are shown in the

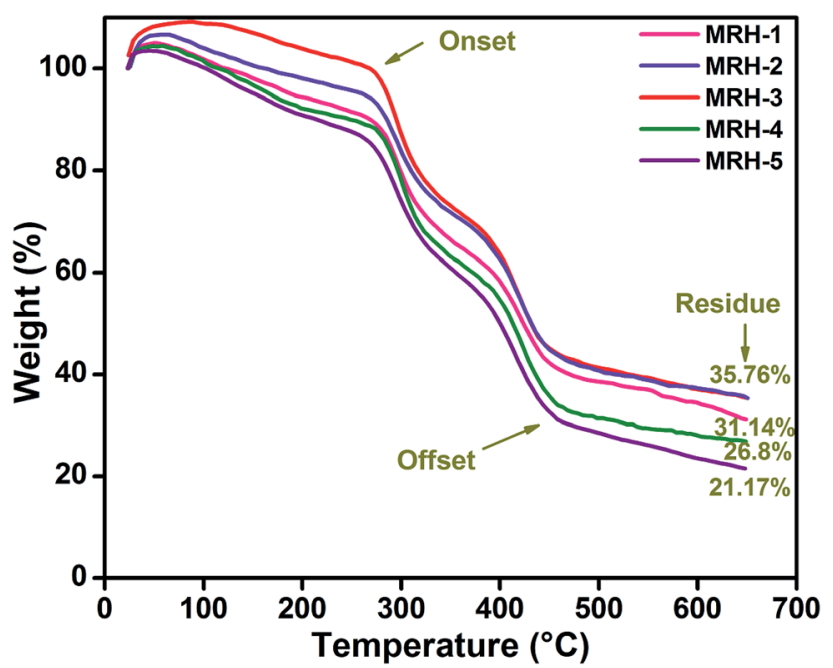

Fig. 8 TGA thermograms of the as-prepared MRHs.

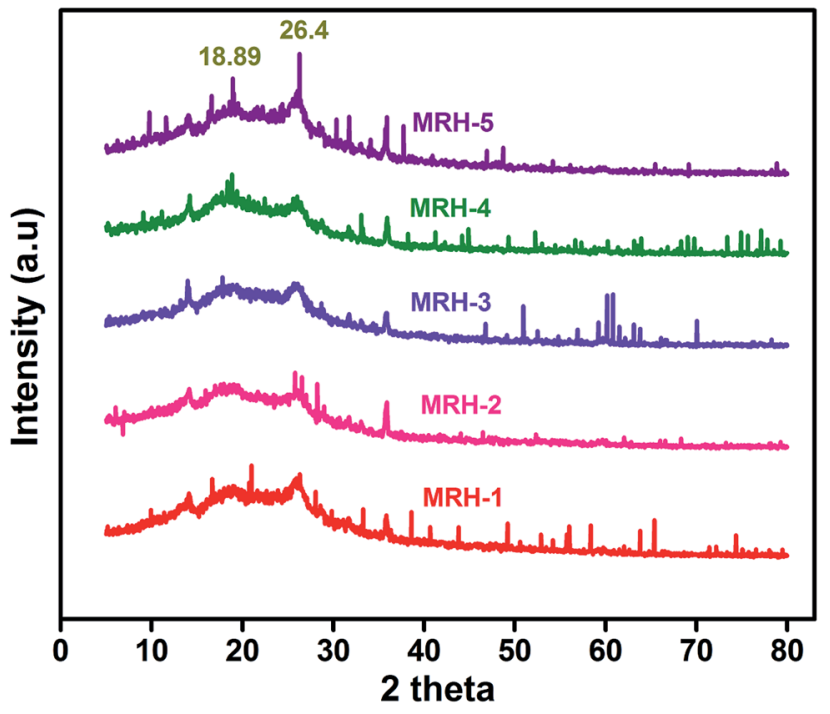

Fig. 9 WAXRD spectra of the as-prepared MRHs.

spectra at $1152 \mathrm{~cm}^{-1}$. The bands at 1288 and $1028 \mathrm{~cm}^{-1}$ are ascribed to the $-\mathrm{C}-\mathrm{O}-\mathrm{C}$ acyclic and cyclic linkages of $\mathrm{CS}$, respectively. The band at $455 \mathrm{~cm}^{-1}$ is ascribed to the $\mathrm{Si}-\mathrm{O}-\mathrm{Si}$ linkages of TEOS, which develops hydrogen bonds between CS and PVP. ${ }^{29}$

\subsection{Swelling behaviour}

4.2.1. Swelling in distilled water. The release response of MRHs is greatly dependent on the swelling index. In fact, the release mechanism of drugs is dependent upon the swelling ratio. The hydrogels started to absorb water when they came in contact with distilled water, and this degree of swelling increased up to a specific time. As shown in Fig. 3, inclusion of TEOS had a significant effect on the swelling response of the studied hydrogel samples. As the concentration of TEOS increased, the swelling response of the hydrogels showed an upward trend. However, this increasing behaviour was observed

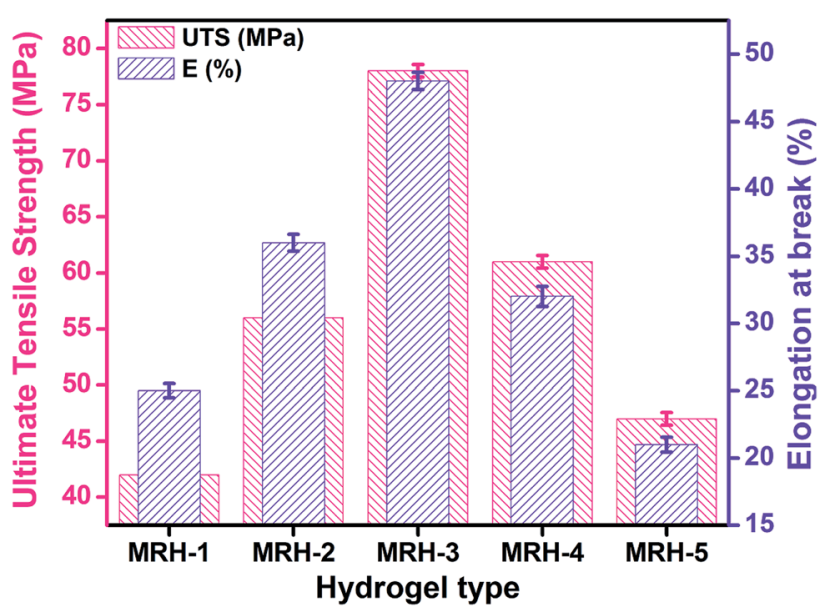

Fig. 10 UTS and E\% of the as-prepared MRHs. 
up to an optimum concentration of TEOS; beyond that, the swelling index was detected to decrease. The first increasing trend is due to the increase of hydrophilicity by increasing the TEOS content, which caused an increase in the number of hydroxyl groups. ${ }^{30}$ The later decreasing behaviour is due to the fact that the high concentration of crosslinker restricted the movement of the CS and PVP chains by developing a compact network, thus hindering the absorption of water into the crosslinked network. ${ }^{31}$

4.2.1.1. Water diffusion mechanism. Generally, the swelling of hydrogel materials depends upon the diffusion mechanism, i.e. the diffusion of water from the extracellular matrix to its structure. The diffusion mechanism can be described by eqn (5):

$$
F=k t^{n}
$$

where $F$ is the fractional swelling determined by the swelling ratio of $W_{\mathrm{t}}$ and $W_{\text {eq }}\left(W_{\mathrm{t}}\right.$ is the weight at time $t$ and $W_{\text {eq }}$ is the weight of the material at the equilibrium time (min), respectively), $k$ is the rate constant and $n$ is the swelling exponent. The swelling data of the MRHs were used to measure the values of $n$ and $k$. The plots of $\ln (F) v s . \ln (t)$ are shown in Fig. 4, and the values of the diffusion parameters are given in Table 1.
It can be observed that the value of $n$ is less than 0.5 , which corresponds to the Fickian transport mechanism; this indicates that the rate of diffusion was lower than the rate of relaxation.

4.2.1.2. Gel fraction. The gel fraction of a hydrogel is the ratio of the dried crosslinked polymer network to the dried hydrogel sample before washing. It can be observed in Table 1 that the gel fractions of the MRHs increased with increasing concentration of crosslinker.

4.2.2. Swelling in $\mathbf{p H}$ solutions. Inspection of Fig. 5 reveals quantitatively different swelling behaviours at different $\mathrm{pH}$ values $(2,4,6,7,8$ and 10). It was observed that at lower $\mathrm{pH}$, the prepared MRHs showed pronounced swelling compared to the swelling in neutral and alkaline conditions. Because PVP is not significantly sensitive to $\mathrm{pH}$, the swelling changes are mainly due to $\mathrm{CS}$ in different $\mathrm{pH}$ solutions. At low $\mathrm{pH}$, the amine groups of CS undergo protonation, which leads to repulsion of the $\mathrm{NH}_{3}{ }^{+}-\mathrm{NH}_{3}{ }^{+}$groups in the polymer chains; this ultimately increases the osmotic pressure inside the network and dissociates secondary interactions such as H-bonding, allowing the absorption of more water into the polymer chains of the MRHs. ${ }^{18,32}$ As the $\mathrm{pH}$ increases towards neutral and alkaline conditions, deprotonation of the amine functional groups takes place and the $\mathrm{NH}_{3}{ }^{+}$groups change back to $\mathrm{NH}_{2}$ moieties; this causes the formation of $\mathrm{H}$-bonds among amino groups, and
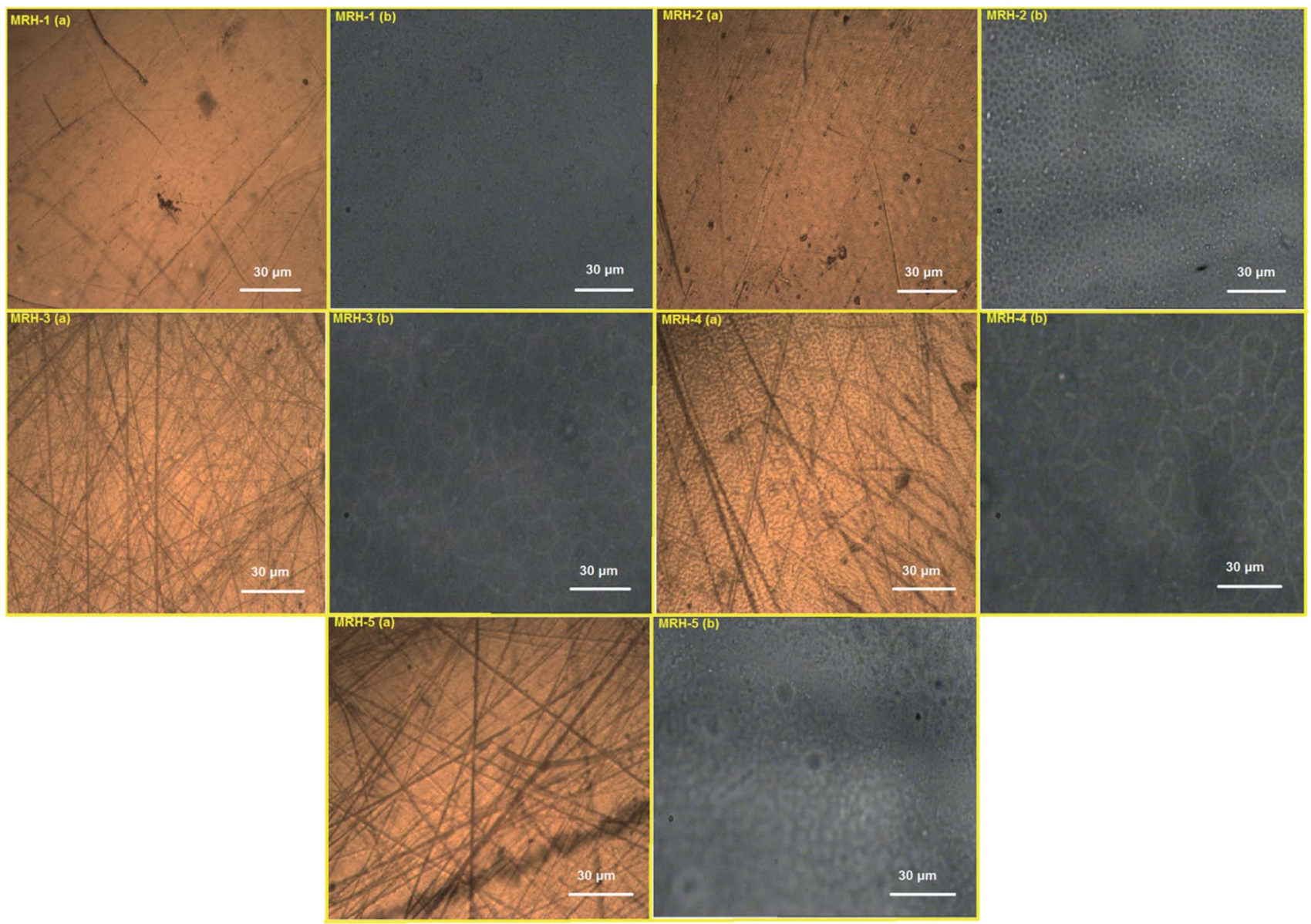

Fig. 11 Optical micrographs of (a) dried and (b) swollen MRHs. 
shrinkage of the repulsion of the polymer chains eventually occurs. ${ }^{33}$

The degrees of swelling of the MRHs depend upon the network structures of the hydrogels, which are determined by the amount of crosslinker. Beyond the optimum concentration of crosslinker, the networks of the MRHs become denser, which leads to a decrease in swelling. ${ }^{34}$ Therefore, in different buffer solutions, MRH-3 showed the maximum swelling; after that concentration, due to the denser networks, the swelling showed a decreasing trend.

4.2.3. Swelling in electrolyte solutions. Hydrogels do not show substantial swelling capacity in electrolyte solutions due to the imbalance of hydrophilic-hydrophobic ions in the network and the ex-osmosis phenomenon. ${ }^{35}$ The swelling indices of the prepared hydrogels in $\mathrm{NaCl}$ and $\mathrm{CaCl}_{2}$ at different concentrations are shown in Fig. 6 and 7, respectively. It was observed that the swelling ratios of the hydrogel samples decreased with increasing concentration of the salt solutions. The shrinkage and swelling behaviour of hydrogels in salt solutions can be determined by ionic interactions between fixed charges and mobile ions, which develop osmotic pressure between the external solutions and the hydrogel samples. At higher concentrations of $\mathrm{NaCl}$ solution, the swelling in the hydrogels decreased due to the increase in osmotic pressure of the solution ions and the decrease in Donnan osmotic pressure. The different swelling behaviour with different crosslinker contents is attributed to changes in the crosslinking density in the hydrogel networks. ${ }^{36,37}$

Fig. 7 shows that the equilibrium swelling of the hydrogels was lower in $\mathrm{CaCl}_{2}$ compared to $\mathrm{NaCl}$ with increasing salt concentration. This result is attributed to the decrease in the osmotic pressure difference between the external solvent and the hydrogel. The charge shielding effect of cations is another parameter that affects the swelling in salt solutions. This effect decreases the anion-anion repulsive forces in the hydrogels. ${ }^{38}$ The swelling of the hydrogels follows this order: $\mathrm{Na}^{+}>\mathrm{Ca}^{2+}$. Another reason is the electrostatic interactions between the

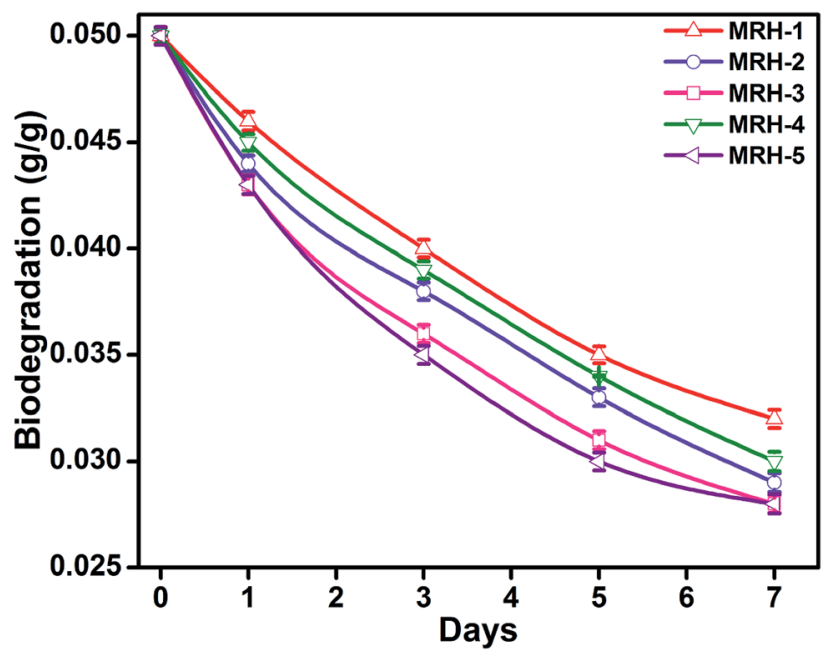

Fig. 12 Biodegradation of the as-prepared MRHs in PBS solution. cationic salt ions and the anionic groups of the polymer chains; this results in an increase in ionic crosslinking, which increases the rigidity and causes deswelling of the hydrogels. ${ }^{39}$

\subsection{Thermogravimetric analysis}

The thermal stability of the MRHs was examined by TGA. This is considered to be the most reliable method to determine the thermal stability of materials. The thermograms of the MRHs showed multiple weight loss stages of the prepared hydrogels (Fig. 8). The first step weight loss from room temperature to $260{ }^{\circ} \mathrm{C}$ is due to the evaporation of moisture, bound water and $\mathrm{H}$-bonded water due to the strongly absorbing nature of CS and PVP. $^{40}$ However, this degradation step was different for the different MRH samples due to the different water absorption capacities of each individual sample. The next stage of weight loss up to $400{ }^{\circ} \mathrm{C}$ is due to the scissoring of interactions between different ingredients and degradation of the ether linkages present in CS. The last stage of weight loss is corresponded to the degradation of the backbone chains of the polymers. ${ }^{41}$ The broadening in the decomposition of all samples indicated the homogeneous blending of CS with PVP, which formed hydrogen bonds between the components. ${ }^{42}$

These thermograms also demonstrated the effects of the crosslinker loading on the thermal stability of the prepared MRHs. It can be observed that the thermal stability of the samples increased dramatically with increasing concentration of TEOS up to MRH-3 (35.76\% residue). When the concentration of TEOS was increased fourfold $(200 \mu \mathrm{L})$, the thermal stability started to decrease $(26.8 \%$ residue). A further increase in TEOS concentration caused a significant decrease in the thermal stability (21.17\% residue). Hence, MRH-3 can be considered as a threshold towards maximum thermal stability of these materials. ${ }^{34}$

\subsection{Wide-angle $X$-ray diffraction analysis}

In order to evaluate the crystalline behaviour of the prepared MRHs, WAXRD was conducted. The WAXRD patterns of the



Fig. 13 Antibacterial activities of the as-prepared MRHs. 
MRHs are shown in Fig. 9. The crystalline peaks at $18.89^{\circ}$ and $26.4^{\circ}$ are due to the semi-crystalline nature of CS. ${ }^{43,44}$ The peaks of PVP appear at approximately the same positions; therefore, both peaks overlap in the same region. ${ }^{45}$ CS shows two crystalline peaks due to the hydroxyl and amino moieties of CS, which are more susceptible to forming strong hydrogen bonds and show crystalline behaviour. The small shifts and broadness of the diffraction peaks are due to the high miscibility and intermolecular interactions between the components. ${ }^{46}$

\subsection{Mechanical properties}

Mechanical integrity is an important property for in vitro and in vivo applications of hydrogels in controlled drug release systems. UTS and $E \%$ were evaluated to examine the mechanical properties of the MRHs, and the results are shown in Fig. 10. For in vivo applications, hydrogels should be pliable enough to endure surrounding tissues and have enough structural integrity to withstand subcutaneous and intraluminal pressure from surrounding structures. ${ }^{47}$ It was observed that UTS and $E \%$ increased gradually up to a TEOS concentration of $150 \mu \mathrm{L}$; beyond that concentration, a decreasing trend was observed. The enhancement of these properties is due to increased interactions and crosslinking between CS, PVP and TEOS because various types of chemical and physical crosslinks exist in the system to develop the network structure. The decreases in the UTS and $E \%$ may be due to the development of more compact network structures, which increase the brittleness and ultimately decrease the mechanical properties of the samples. The improved fracture strain and toughness can enhance their performance in novel biomedical applications. ${ }^{48}$

\subsection{Optical microscopy}

In the microscopy of hydrogels, it is imperative that the hydrogels are in a hydrated form to ensure accurate representation of their swollen structures. Commonly used microscopic techniques such as scanning electron microscopy and transmission electron microscopy result in changes in the microstructures and crystallinity of hydrogels due to drying during microscopic analysis. ${ }^{49}$ To restrain the effects of drying, prepared MRHs in dry and swollen forms were analyzed by an optical microscope. The brown images in Fig. 11 show micrographs of the dried MRHs, while the respective black images show micrographs of the swollen samples. It can be observed that with increasing concentration of crosslinker, a more compacted network structure appears. In the swollen micrographs, the pockets or pore sizes increase up to the MRH-3 sample; however, beyond that concentration, a declining trend was observed. This behaviour of the MRHs is due to the decrease in the distance between the polymer chains, the increase in hydrogen bonding, and crystallite formation. ${ }^{\mathbf{5 0}}$ This result is in accordance with the behaviour noted for the swelling indices in water.

\subsection{In vitro biodegradation analysis}

The degradability of a material determines its mode of application, and the degradation behaviour of the hydrogels was determined with respect to time by weight loss measurements. The MRHs showed downward trends with time (Fig. 12); this degradation is due to the fact that CS degrades in PBS solution at a slow rate. The connectivity of the CS-based hydrogel network is linked with $\mathrm{C}=\mathrm{C}$ bonds, which yield alkyl linkages. Hence, the degradation of the hydrogel samples is due to dissociation of the glycosidic bonds of the CS molecules. ${ }^{51}$ The graph shows that the hydrogels degraded consistently with time $(14.28 \%$ from MRH-1 to MRH-3; the degradation extent was lower at higher concentrations of crosslinker). This lower degradation extent at higher loadings of TEOS was ascribed to the higher crosslinking densities and the compact networks of the hydrogels. This increased compact network of the material is responsible for absorbing a lower amount of water, which can assist the degradation. These results are in agreement with those of McBath and Ship. ${ }^{52}$

\subsection{Antimicrobial activity}

The antibacterial activities of the MRHs were determined using E. coli by implementing the JIS L 1902-2002 test to evaluate the resistance of bacterial growth. The antibacterial activities of the prepared MRHs are shown in Fig. 13 in terms of optical density (OD). The OD of the control sample (without hydrogel sample) was observed as 1.287, while those of the other samples containing hydrogels were lower; this provides evidence of the antibacterial properties of the hydrogels against $E$. coli. The lowest OD value is that of MRH-3; after that optimum concentration of crosslinker, the OD values are slightly high. The inclusion of hydrophilic crosslinker moieties (silanol groups of TEOS) in the hydrogels allowed the formation of hydrophilic hydrogels with good swelling indices in aqueous and organic media and with greater positive charge densities. The $\mathrm{C}=\mathrm{O}$ and NH- moieties in PVP and CS present in the MRHs could be protonated and the net positive charge density was eventually strengthened, which led to better antibacterial activity. ${ }^{53}$

\subsection{Cytotoxicity analysis}

In vitro cytotoxicity analysis provides insight into potential issues with local tissue response. One method to determine cell and tissue response is to evaluate the cytotoxicity, or the level of toxicity to cells. Cytotoxicity can be determined by the inhibition of cell proliferation or cell lysis. ${ }^{54}$ In vitro cytotoxicity analysis of the prepared hydrogel samples was performed by counting live and dead nauplii by light microscope and then applying the formula described in Section 3.7. Deaths of nauplii may be due

Table 2 Percent mortality of $A$. salina after $24 \mathrm{~h}$ exposure to the MRHs

\begin{tabular}{ll}
\hline Type of hydrogel & Morta \\
\hline MRH-1 & 2.35 \\
MRH-2 & 2.44 \\
MRH-3 & 2.05 \\
MRH-4 & 2.34 \\
MRH-5 & 2.67
\end{tabular}



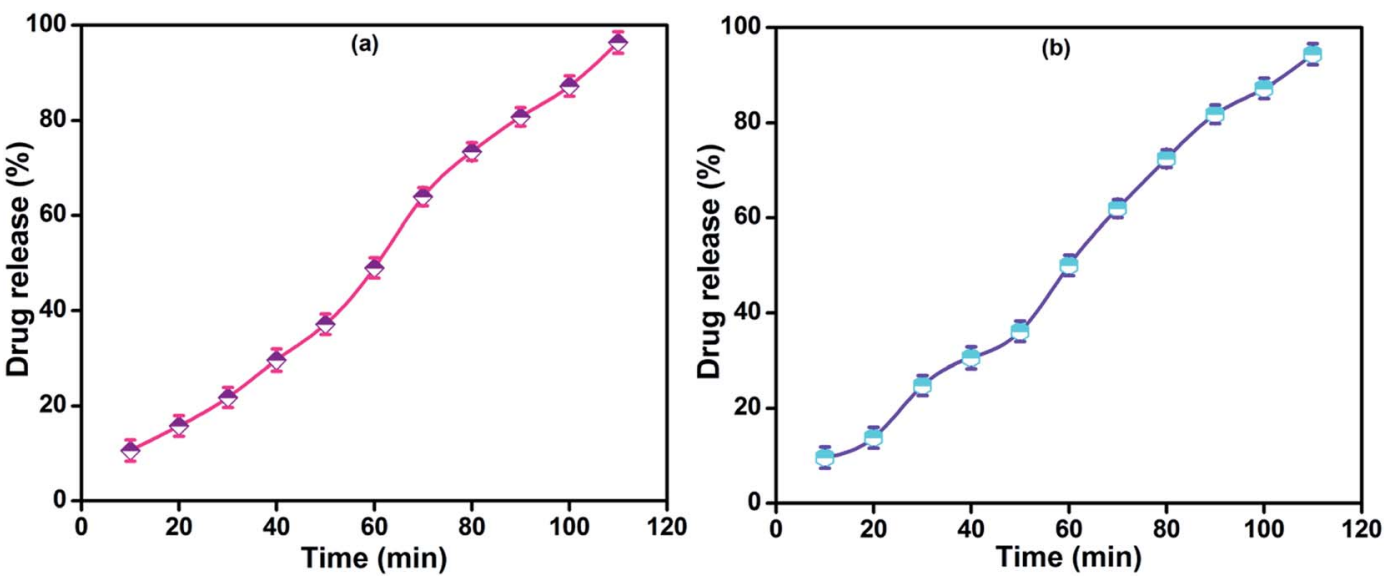

Fig. 14 Drug release profiles of $\mathrm{MRH}-3$ in (a) PBS solution $(\mathrm{pH} 7.4)$ and (b) SIF solution (pH 6.8) at $37^{\circ} \mathrm{C}$.

to three reasons: cytotoxicity of chemicals, deficiency of dissolved oxygen in sea water and development of a viscous layer on the gills of the nauplii. ${ }^{27}$ In this work, the possibility of the death of the nauplii due to toxicity is very low because the percent mortality is very low at lower concentrations of crosslinker (Table 2). Moreover, these hydrogel samples were prepared by CS, PVP and TEOS, and all these ingredients are biocompatible and approved by the FDA. The low percent mortality of the hydrogel samples may be due to the formation of viscous layers on the gills of the nauplii; this eventually inhibits the oxygen permeability and causes the deaths of nauplii and an increase in the percent mortality beyond the optimum concentration $(150 \mu \mathrm{L})$ of TEOS. ${ }^{55}$

\subsection{Drug release profiles}

4.10.1. Drug release profiles in PBS and SIF solutions. Based upon the mechanisms of drug release from the polymer network, drugs are released by four different mechanisms, which are stimulated release, degradation-controlled, solventcontrolled and diffusion-controlled. The solvent-controlled mechanism is further classified into osmotic pressure- controlled and swelling-controlled mechanisms. ${ }^{56}$ The drug release behaviour of the prepared hydrogels is strongly linked to the swelling characteristics of the hydrogels, which is a key parameter of the structural design of hydrogels. When a hydrogel undergoes swelling, its mesh size increases, and drug release to the surface of the device occurs through the diffusion pathways. ${ }^{57,58}$

In vitro drug release of lidocaine from MRH-3 was evaluated in SGF, SIF and PBS solutions as a function of time, as shown in Fig. 14. In SGF solution, the lidocaine was completely released in less than $30 \mathrm{~min}$; this is not in accordance with the standard pharmacopeia, according to which the release of a drug must not be greater than $10 \%$ in SGF solution. This release profile shows that $96.37 \%$ lidocaine was released in a controlled way in 110 min in PBS (pH 7.4) solution; whereas in SIF, 94.37\% lidocaine was released in $110 \mathrm{~min}$, which is in accord with the US pharmacopeia standard (USP XXIV). ${ }^{59}$

At pH values close to neutral, such as 6.8 and 7.4, the amino groups of CS are not protonated, which causes development of the physical networks in the hydrogels. The resulting network is responsible for the controlled release of lidocaine in PBS
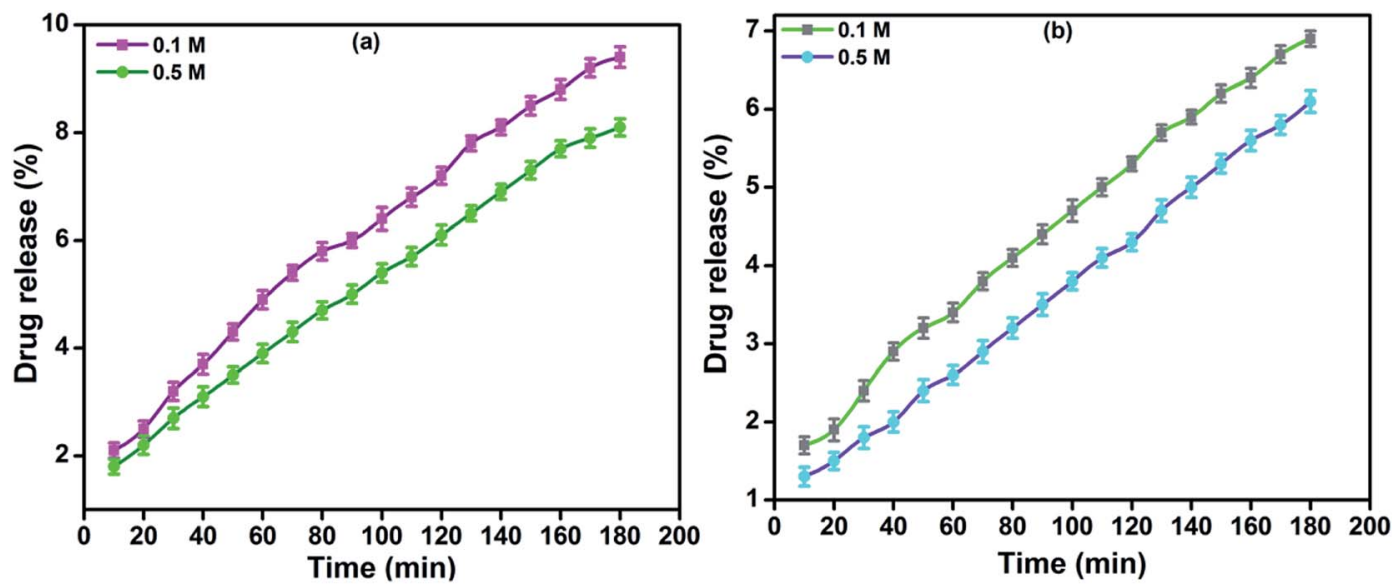

Fig. 15 Drug release profiles of $\mathrm{MRH}-3$ in (a) $\mathrm{NaCl}$ and (b) $\mathrm{CaCl}_{2}$ solutions. 
solution. ${ }^{62}$ The remaining amount of drug could not be easily determined because the hydrogel film was broken into tiny pieces. Butt et al. developed a hydrogel using CS and determined its drug release behaviour in PBS, SGF and SIF solutions. ${ }^{60}$ The results indicated that the prepared hydrogel can be used for controlled drug delivery systems and other biomedical applications.

4.10.2. Drug release in electrolyte solutions. Generally, as the concentration of electrolyte solution increases, the polymer solubility decreases and, ultimately, the drug release rate slows. The amount of water available to hydrate the polymer, which ultimately releases the drug after swelling, will decrease because more water is required to maintain the ions in electrolyte solutions. The type of ion also affects the extent of polymer hydration. ${ }^{61}$

The in vitro drug release profiles of $\mathrm{MRH}-3$ in $\mathrm{NaCl}$ and $\mathrm{CaCl}_{2}$ solutions as a function of time are shown in Fig. 15. It was observed that the lidocaine release was slower in $\mathrm{CaCl}_{2}$ solutions than in $\mathrm{NaCl}$ solutions at higher concentrations of the electrolytes. At a lower concentration $(0.1 \mathrm{M})$ of electrolyte, the polymer chains swell and allow the drug to be released; whereas, at a higher concentration $(0.5 \mathrm{M})$, less water is available to swell the polymer chains, and the rate of drug release was therefore more slow.

\section{Conclusions}

A novel multi-responsive series of hydrogels which are capable of prolonging the release of lidocaine were successfully devised in this study by a solution casting approach. These prepared hydrogels contain complex networks of CS, PVP and TEOS and have sufficient mechanical integrity, i.e. UTS of $78 \mathrm{MPa}$ and $E \%$ of 48 , to carry the drug through the body. The physical and chemical intermolecular interactions among all the components of the hydrogels were confirmed by FTIR spectroscopy. The prepared hydrogels showed the highest swelling indices $\left(140.75 \mathrm{~g} \mathrm{~g}^{-1}\right)$ in distilled water, at acidic $\mathrm{pH}(\mathrm{pH} 4)$ and in electrolyte solutions at the optimum concentration $(150 \mu \mathrm{L})$ of crosslinker. The hydrogel sample with the same concentration of TEOS showed the highest thermal stability (35\%) as evaluated by TGA, and its crystalline behaviour was assessed by WXRD. Optical microscopy of dried and swollen MRH samples was also conducted to evaluate their porous and networked structures. On the basis of their biodegradability, antimicrobial activity and cytotoxicity analyses, we envision that our MRHs will find potential applications in drug delivery. To validate the significance of the prepared MRHs, controlled release (96.14 and $94.37 \%$ ) of lidocaine-loaded MRH-3 was obtained in PBS and SIF solutions, respectively, over a period of $110 \mathrm{~min}$; thus, this hydrogel is a potential candidate for use in in vivo drug release applications.

\section{Conflicts of interest}

There are no conflicts to declare.

\section{Acknowledgements}

We express our cordial gratitude to Prof. Dr Javed Iqbal Qazi, Miss Shamsa Jabeen and Miss Farah Deeba, Department of Zoology, University of the Punjab, Lahore, Pakistan for assistance in the antimicrobial analysis, Mr Mohsin Cheema, Department of MicroBiology and Molecular Genetics, University of the Punjab, Lahore, Pakistan, for cytotoxicity analysis, and Miss Farheen Anjum, Centre for Undergraduate Studies, University of the Punjab, Lahore, Pakistan for drug release analysis of our prepared samples. We would like to extend our deep gratitude to Prof. Dr Tahir Jamil (late) for his patient guidance, enthusiastic encouragement and useful critiques for this research work.

\section{References}

1 M. V. Risbud, A. A. Hardikar, S. V. Bhat and R. R. Bhonde, $\mathrm{pH}$-sensitive freeze-dried chitosan-polyvinyl pyrrolidone hydrogels as controlled release system for antibiotic delivery, J. Controlled Release, 2000, 68, 23-30.

2 I. G. Needleman, F. C. Smales and G. P. Martin, An investigation of bioadhesion for periodontal and oral mucosal drug delivery, J. Clin. Periodontol., 1997, 24, 394400.

3 A. A. Deshpande, N. H. Shah, C. T. Rhodes and W. Malick, Development of a novel controlled-release system for gastric retention, Pharm. Res., 1997, 14, 815-819.

4 R. P. Hari, T. Chandy and C. P. Sharma, Chitosan/calcium alginate microcapsules for intestinal delivery of nitrofurantoin, J. Microencapsulation, 1996, 13, 319-329.

5 M. G. Cascone, B. Sim and S. Downes, Blends of synthetic and natural polymers as drug delivery systems for growth hormone, Biomaterials, 1995, 16, 569-574.

6 M. V. Risbud and S. V. Bhat, Properties of polyvinyl pyrrolidone/b-chitosan hydrogel membranes and their biocompatibility evaluation by haemorheological method, J. Mater. Sci.: Mater. Med., 2001, 12, 75-79.

7 S. A. Dergunov, I. K. Nam, G. A. Mun, Z. S. Nurkeeva and E. M. Shaikhutdinov, Radiation synthesis and characterization of stimuli sensitive chitosan-polyvinyl pyrollidone hydrogels, Radiat. Phys. Chem., 2005, 72, 619623.

8 S. Dumitriu, M. Popa and M. Dumitriu, Review : Polymeric biomaterials as enzyme and drug carriers* Part III: Polymeric drugs and drug delivery systems, J. Bioact. Compat. Polym., 1989, 4, 57-73.

9 Y. C. Nho and K. R. Park, Preparation and properties of PVA/ PVP hydrogels containing chitosan by radiation, J. Appl. Polym. Sci., 2001, 85, 1787-1794.

10 M. Kawase, N. Michibayashi, Y. Nakashima, N. Kurikawa, K. Yagi and T. Mizoguchi, Application of glutaraldehydecrosslinked chitosan as a scaffold for hepatocyte attachment, Biol. Pharm. Bull., 1997, 20, 708-710.

11 N. A. Peppas and A. S. Hoffman, Biomaterials Science: An Introduction to Materials in Medicine, Academic Press, New York, London, 1996, p. 1573. 
12 K. R. Park and Y. C. Nho, Synthesis of PVA/PVP hydrogels having two-layer by radiation and their physical properties, Radiat. Phys. Chem., 2003, 67, 361-365.

13 W. Wang, Y. Du, Y. Qiu, X. Wang, Y. Hu, J. Yang, J. Cai and J. F. Kennedy, A new green technology for direct production of low molecular weight chitosan, Carbohydr. Polym., 2008, 74, 127-132.

14 G. Ma, D. Yang, Y. Zhou, M. Xiao, J. F. Kennedy and J. Nie, Preparation and characterization of water-soluble Nalkylated chitosan, Carbohydr. Polym., 2008, 74, 121-126.

15 G. Ma, D. Yang, D. Su, X. Mu, J. F. Kennedy and J. Nie, Preparation and properties of water-soluble chitosan and polyvinyl alcohol blend films as potential bone tissue engineering matrix, Polym. Adv. Technol., 2009, 21, 189-195.

16 J. Xi, G. Yuan, L. Kong, Y. Gong, N. Zhao and X. Zhang, Behavior of MC3T3-E1 Osteoblast cultured on chitosan modified with polyvinylpyrrolidone, Tsinghua Sci. Technol., 2005, 10, 439-444.

$17 \mathrm{H}$. Kaplan and A. Güner, Characterization and determination of swelling and diffusion characteristics of poly(n-vinyl-2-pyrrolidone) hydrogels in water, J. Appl. Polym. Sci., 2000, 78, 994-1000.

18 J. R. Khurma, D. R. Rohindra and A. V. Nand, Swelling and thermal characteristics of genipin crosslinked chitosan and poly(vinyl pyrrolidone) hydrogels, Polym. Bull., 2005, 54, 195-204.

19 E. Marsano, S. Vicini, J. Skopinska, M. Wisniewski and A. Sionkowska, Chitosan and poly(vinyl pyrrolidone): Compatibility and miscibility of blends, Macromol. Symp., 2004, 218, 251-260.

20 B. Ballantyne and S. L. Jordan, Toxicological, medical and industrial hygiene aspects of glutaraldehyde with particular reference to its biocidal use in cold sterilization procedures, J. Appl. Toxicol., 2001, 21, 131-151.

21 S. Liang, L. Liu, Q. Huang and K. L. Yam, Preparation of single or double - network chitosan/poly(vinyl alcohol) gel films through selectively cross-linking method, Carbohydr. Polym., 2009, 77, 718-724.

22 K. K. Jena and K. V. S. N. Raju, Synthesis and characterization of hyperbranched polyurethane hybrids using tetraethoxysilane (TEOS) as cross-linker, Ind. Eng. Chem. Res., 2008, 47, 9214-9224.

23 Q. Hou, P. A. De Bank and K. M. Shakesheff, Injectable scaffolds for tissue regeneration, J. Mater. Chem., 2004, 14, 1915-1923.

$24 \mathrm{~S}$. Y. Lee and G. Tae, Formulation and in vitro characterization of an in situ gelable, photo-polymerizable Pluronic hydrogel suitable for injection, J. Controlled Release, 2007, 119, 313-319.

25 B. K. Kumar, V. S. T. Rajan and N. T. Begum, Analytical method development and validation of Lidocaine in ointment formulation by U.V spectrophotometric method, Int. J. Pharm. Pharm. Sci., 2012, 4, 610-614.

26 L. Neufeld and H. Bianco-Peled, Pectin-chitosan physical hydrogels as potential drug delivery vehicles, Int. J. Biol. Macromol., 2017, 101, 852-861.
27 M. N. Khan, J. M. M. Islam and M. A. Khan, Fabrication and characterization of gelatin-based biocompatible porous composite scaffold for bone tissue engineering, J. Biomed. Mater. Res., Part A, 2012, 100A, 3020-3028.

28 D.-G. Yu, G. R. Williams, J.-H. Yang, X. Wang, W. Qian and Y. Li, Chitosan nanoparticles self-assembled from electrospun composite nanofibers, J. Text. Sci. Eng., 2012, 2, 1-5.

29 E. F. dos Reis, F. S. Campos, A. P. Lage, R. C. Leite, L. G. Heneine, W. L. Vasconcelos, Z. I. P. Lobato and H. S. Mansur, Synthesis and characterization of poly (vinyl alcohol) hydrogels and hybrids for rMPB70 protein adsorption, Mater. Res., 2006, 9, 185-191.

30 W. Wei, J. Li, X. Qi, Z. Yin, G. Zuo, X. Pan, T. Su, J. Zhang and W. Dong, Synthesis and characterization of a multi-sensitive polysaccharide hydrogel for drug delivery, Carbohydr. Polym., 2017, 177, 275-283.

31 S. M. H. Dabiri, A. Lagazzo, F. Barberis, A. Shayganpour, E. Finocchio and L. Pastorino, New in situ synthetized hydrogel composite based on alginate and brushite as a potential $\mathrm{pH}$ sensitive drug delivery system, Carbohydr. Polym., 2017, 177, 324-333.

32 H. Ferfera-Harrar, N. Aiouaz and N. Dairi, Synthesis and properties of chitosan-graft polyacrylamide/gelatin superabsorbent composites for wastewater purification, International Journal of Chemical and Molecular Engineering, 2015, 9, 849-856.

33 M. C. F. C. Felinto, D. F. Parra, C. C. da Silva, J. Angerami, M. J. A. Oliveira and A. B. Lugão, The swelling behavior of chitosan hydrogels membranes obtained by UV- and cradiation, Nucl. Instrum. Methods Phys. Res., Sect. B, 2007, 265, 418-424.

34 S. Ekici and D. Saraydin, Interpenetrating polymeric network hydrogels for potential gastrointestinal drug release, Polym. Int., 2007, 56, 1371-1377.

35 S. Nesrinne and A. Djamel, Synthesis, characterization and rheological behavior of $\mathrm{pH}$ sensitive poly(acrylamide-coacrylic acid) hydrogels, Arabian J. Chem., 2017, 10, 539-547.

36 C. Chang, M. He, J. Zhou and L. Zhang, Swelling behaviors of pH- and salt-responsive cellulose-based hydrogels, Macromolecules, 2011, 44, 1642-1648.

37 L. Zhao, L. Xu, H. Mitomo and F. Yoshii, Synthesis of pHsensitive PVP/CM-chitosan hydrogels with improved surface property by irradiation, Carbohydr. Polym., 2006, 64, 473-480.

38 J. Yu, G. Yang, L. Yun, Y. Wu, J. Gao and Q. Lu, Synthesis, Characterization, and swelling behaviors of acrylic acid/ carboxymethyl cellulose superabsorbent hydrogel by glowdischarge electrolysis plasma, Polym. Eng. Sci., 2014, 23102320.

39 S. Distantina, F. Fadilah and M. Kaavessina, Swelling behaviour of Kappa carrageenan hydrogel in neutral salt solution, International Journal of Chemical and Molecular Engineering, 2016, 10, 998-1001.

40 J.-T. Yeh, C.-L. Chen, K. S. Huang, Y. H. Nien, J. L. Chen and P. Z. Huang, Synthesis, characterization, and application of 
PVP/chitosan blended polymers, J. Appl. Polym. Sci., 2006, 101, 885-891.

41 D. Archana, B. K. Singh, J. Dutta and P. K. Dutta, In vivo evaluation of chitosan-PVP-titanium dioxide nanocomposite as wound dressing material, Carbohydr. Polym., 2013, 95, 530-539.

42 D. Archana, B. K. Singh, J. Dutta and P. K. Dutta, ChitosanPVP-nano silver oxide wound dressing: In vitro and in vivo evaluation, Int. J. Biol. Macromol., 2015, 73, 49-57.

43 S. S. Vaghani and M. M. Patel, Hydrogels based on interpenetrating network of chitosan and polyvinyl pyrrolidone for $\mathrm{pH}$-sensitive delivery of repaglinide, Curr. Drug Discovery Technol., 2011, 8, 126-135.

44 S. S. Vaghani and M. M. Patel, pH-sensitive hydrogels based on semi-interpenetrating network (semi-IPN) of chitosan and polyvinyl pyrrolidone for clarithromycin release, Drug Dev. Ind. Pharm., 2011, 37, 1160-1169.

45 R. K. Mishra, S. Mondal, M. Datt and A. K. Banthia, Development and characterization of chitosan and phosphomolybdic acid (PMA) based composites, Int. J. Plast. Technol., 2010, 14, 80-92.

46 R. Poonguzhali, S. K. Basha and V. Sugantha Kumari, Synthesis and characterization of chitosan/ poly(vinylpyrrolidone) biocomposite for biomedical application, Polym. Bull., 2017, 74, 2185-2204.

47 X. Ma, X. Sun, D. Hargrove, J. Chen, D. Song, Q. Dong, X. Lu, T.-H. Fan, Y. Fu and Y. Lei, A biocompatible and biodegradable protein hydrogel with green and red autofluorescence: preparation, characterization and in vivo biodegradation tracking and modeling, Sci. Rep., 2016, 6, 19370.

48 A. Islam, T. Yasin, N. Gull, S. M. Khan, A. Sabir, M. A. Munawwar, M. Shafiq, T. Jamil and M. H. Raza, Fabrication and performance characteristics of tough hydrogel scaffolds based on biocompatible polymers, Int. J. Biol. Macromol., 2016, 92, 1-10.

49 J. L. Holloway, A. M. Lowman and G. R. Palmese, Aging behavior of PVA hydrogels for soft tissue applications after in vitro swelling using osmotic pressure solutions, Acta Biomater., 2013, 9, 5013-5021.

50 J. L. Holloway, K. L. Spiller, A. M. Lowman and G. R. Palmese, Analysis of the in vitro swelling behavior of poly(vinyl alcohol) hydrogels in osmotic pressure solution for soft tissue replacement, Acta Biomater., 2011, 7, 24772482 .
51 Y. Hong, H. Song, Y. Gong, Z. Mao, C. Gao and J. Shen, Covalently crosslinked chitosan hydrogel: Properties of in vitro degradation and chondrocyte encapsulation, Acta Biomater., 2007, 3, 23-31.

52 R. A. McBath and D. A. Shipp, Swelling and degradation of hydrogels synthesized with degradable poly(b-amino ester) crosslinkers, Polym. Chem., 2010, 1, 860-865.

53 N. A. Mohamed and M. M. Fahmy, Synthesis and antimicrobial activity of some novel cross-linked chitosan hydrogels, Int. J. Mol. Sci., 2012, 13, 11194-11209.

54 M. M. Rahman, S. Afrin and P. Haque, Characterization of crystalline cellulose of jute reinforced poly(vinyl alcohol) (PVA) biocomposite film for potential biomedical applications, Prog. Biomater., 2014, 3, 23.

55 M. F. Rahman, J. M. M. Islam, M. M. Hassan, S. M. A. Habib, M. S. Pervez and M. A. Khan, Development of urea crosslinked thin film for biomedical application, Int. J. Polym. Mater. Polym. Biomater., 2013, 62, 695-699.

56 G.-H. Son, B.-J. Lee and C.-W. Cho, Mechanisms of drug release from advanced drug formulations such as polymeric-based drug-delivery systems and lipid nanoparticles, J. Pharm. Invest., 2017, 47, 287-296.

57 M. F. Akhtar, N. M. Ranjha and M. Hanif, Effect of ethylene glycol dimethacrylate on swelling and on metformin hydrochloride release behavior of chemically crosslinked pH-sensitive acrylic acid-polyvinyl alcohol hydrogel, Daru, J. Pharm. Sci., 2015, 23, 41-50.

$58 \mathrm{~J}$. Li and D. J. Mooney, Designing hydrogels for controlled drug delivery, Nat. Rev. Mater., 2016, 12, 1-38.

59 A. Islam and T. Yasin, Controlled delivery of drug from $\mathrm{pH}$ sensitive chitosan/poly (vinyl alcohol) blend, Carbohydr. Polym., 2012, 88, 1055-1060.

60 A. Butt, S. Jabeen, N. Nisar, A. Islam, N. Gull, S. S. Iqbal, S. M. Khan and B. Yameen, Controlled release of cephradine by biopolymers based target specific crosslinked hydrogels, Int. J. Biol. Macromol., 2019, 121, 104-112.

$61 \mathrm{~J}$. L. Johnson, J. Holinej and M. D. Williams, Influence of ionic strength on matrix integrity and drug release from hydroxypropyl cellulose compacts, Int. J. Pharm., 1993, 90, 151-159.

62 N. Gull, S. M. Khan, M. T. Z. Butt, S. Zia, S. Khalid, A. Islam, I. Sajid, R. U. Khan and M. W. King, Hybrid coss-linked hydrogels as a technology platform for in vitro release of cephradine, Polym. Adv. Technol, 2019, 30, 2414-2424. 